\title{
Paper Microzone Plates as Analytical Tools for Studying Enzyme Stability: A Case Study on the Stabilization of Horseradish Peroxidase Using Trehalose and SU-8 Epoxy Novolac Resin
}

\author{
Kirsten A. Ganaja, ${ }^{\dagger}$ Cory A. Chaplan, ${ }^{\dagger}$ Jingyi Zhang, $^{\dagger \dagger}$ Nathaniel W. Martinez, ${ }^{\S}$
} and Andres W. Martinez ${ }^{*}{ }^{\dagger}$

${ }^{\dagger}$ Department of Chemistry \& Biochemistry, ${ }^{\S}$ Department of Biological Sciences, California Polytechnic State University, San Luis Obispo, California 93407, United States

${ }^{\ddagger}$ St. Paul's School, Concord, New Hampshire 03301, United States

Supporting Information

ABSTRACT: Paper microzone plates in combination with a noncontact liquid handling robot were demonstrated as tools for studying the stability of enzymes stored on paper. The effect of trehalose and SU-8 epoxy novolac resin (SU-8) on the stability of horseradish peroxidase (HRP) was studied in both a short-term experiment, where the activity of various concentrations of HRP dried on paper were measured after $1 \mathrm{~h}$, and a long-term experiment, where the activity of a single concentration of HRP dried and stored on paper was monitored for 61 days. SU-8 was found to stabilize HRP up to 35 times more than trehalose in the short-term experiment for comparable concentrations of the two reagents, and a $1 \%$ SU- 8 solution was found to stabilize HRP approximately 2 times more than a $34 \%$ trehalose solution in both short- and long-term experiments. The results suggest that SU-8 is a promising candidate for use as an enzyme-stabilizing reagent for paper-based diagnostic devices and that the short-term experiment could be used to quickly evaluate the capacity of various reagents for stabilizing enzymes to identify and characterize new enzyme-stabilizing reagents.
$\mathrm{T}$ he stability and stabilization of enzymes is important for many industrial applications, commercial products, and laboratory protocols that rely on enzymes for catalyzing chemical reactions. ${ }^{1-4}$ For this reason, a vast amount of research has focused on studying the stability of enzymes under various conditions and on developing techniques for stabilizing enzymes. Among these enzyme-stabilizing techniques, the immobilization of enzymes on solid substrates and the use of enzyme-stabilizing reagents, such as trehalose, are two common approaches. $^{3-8}$ Our particular interest in the stability of enzymes is in the context of their use for point-of-care diagnostic devices and, more specifically, for paper-based microfluidic devices. ${ }^{9-13}$

Paper-based microfluidic devices are being explored as platforms for very low-cost point-of-care sensors that could be used in the most remote parts of the world to detect analytes and diagnose disease. ${ }^{11-13}$ For these devices to fulfill this objective, they must be stable under ambient conditions for extended periods of time so that they can be transported into the field without relying on a cold chain. ${ }^{14}$ The development of methods for stabilizing reagents, including enzymes, on paperbased devices is, therefore, a critical component of the development of paper-based sensors. Enzymes used for performing paper-based assays are typically dried on the devices and then rehydrated when the devices are used. ${ }^{15}$ While some publications have explored the stability of enzymatic assays in the context of paper-based microfluidic devices, $8,15,16$ relatively little is known about the stability of enzymes dried on paper. In this article, we describe the use of paper microzone plates as tools for studying the stability of enzymes on paper and for quantifying the effects of enzyme-stabilizing reagents. As a case study, we have investigated the stabilization of horseradish peroxidase (HRP) using trehalose and SU-8 epoxy novolac resin (SU-8) on cellulose-based chromatography paper (Figure 1).

Paper has been used extensively as a solid support for preparing arrays of spots for collecting samples, synthesizing compounds, and performing assays. ${ }^{17-20}$ Paper microzone plates, which differ from other paper-based arrays in that the paper is patterned with a hydrophobic ink to define hydrophilic microzones in the same layout as conventional plastic-based 96well or 384-well plates, were first described by Carrilho et al. in $2009 .^{21}$ The advantages of paper microzone plates are that they can be used to perform large numbers of tests in parallel, the microzones can be filled with small volumes of sample (typically $1-3 \mu \mathrm{L}$ ), and the plates are compatible with all of

Received: December 25, 2016

Accepted: April 24, 2017

Published: May 4, 2017 

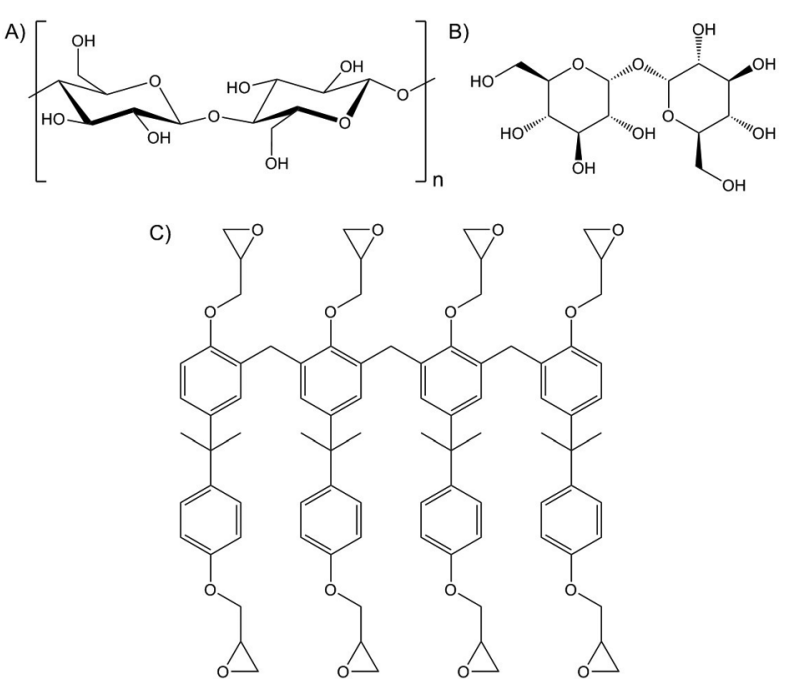

Figure 1. Chemical structures of cellulose (A), trehalose (B), and SU8 (C).

the equipment designed for use with plastic well plates such as plate readers, multichannel pipets, and liquid handling robots. ${ }^{21}$ Therefore, we felt that paper microzone plates would be an ideal platform for studying the stability of enzymes on paper, given that these studies typically require a large number of tests.

HRP was selected as the model enzyme for our study because it is used commonly as a reporter enzyme in many biochemical assays and has already been used extensively in paper-based microfluidic devices. ${ }^{8,13,22}$ HRP catalyzes the oxidation of a wide variety of electron donor substrates by hydrogen peroxide. ${ }^{22}$ When performing an enzymatic assay, one can choose an electron donor substrate that undergoes a detectable change upon oxidation so that a readout signal is produced. For example, chromogenic reagents, such as 2,2'azinobis [3-ethylbenzthiazoline-6-sulfonic acid] diammonium salt (ABTS), that change color upon oxidation are commonly used for biochemical assays involving HRP. ABTS changes from a colorless starting material to a green product upon oxidation and has been used successfully as a substrate for HRP on paper-based devices. ${ }^{16,23}$

The stability of HRP dried on paper-based microfluidic devices has been described in three publications that we are aware of. The first report looked at the stability of a mixture of HRP and glucose oxidase (GOx) dried on paper-based devices for performing a glucose assay. ${ }^{15}$ The devices in this study were stored under ambient conditions in a Ziploc bag for 60 days. The study found that the signal from the assay decreased linearly over time with a half-life of 11 days and that when the enzymes were dried in the presence of $10 \% \mathrm{w} / \mathrm{v}$ trehalose, the signal from the assay was stable for 30 days and then decreased linearly with a half-life that we estimate to be on the order of 75 days (the exact half-life was not reported in the publication). The second report looked at the stability of HRP-conjugated antibodies dried on glass fiber pads in the presence of $0.1 \% \mathrm{w} / \mathrm{v}$ bovine serum albumin (BSA), $4 \% \mathrm{w} / \mathrm{v}$ trehalose, and $0.01 \mathrm{M}$ Fe-EDTA. ${ }^{8}$ The glass fiber pads were vacuum sealed in plastic bags along with a desiccant and stored at $45{ }^{\circ} \mathrm{C}$. Under these conditions, HRP retained $\sim 80 \%$ of its initial activity after 5 months of storage. The third report, conducted by our group, looked at the stability of HRP dried on paper both with and without $10 \% \mathrm{w} / \mathrm{v}$ trehalose and stored wrapped in aluminum foil under ambient conditions. ${ }^{16}$ We found that the signal from the HRP assay had a half-life of 0.4 days in the absence of trehalose and a half-life of 1.1 days in the presence of trehalose.

All three studies confirmed the enzyme-stabilizing effect of trehalose, an alpha-linked disaccharide that is known to stabilize enzymes and has been studied extensively for this purpose. ${ }^{5}$ Although we recognize that the experimental setup and storage conditions from the first and third studies were different, the difference in the half-lives for the signals of the enzymatic assays (75 days in the first study versus 1.1 days in the third study, both in the presence of $10 \%$ trehalose) was so large that it suggested that another enzyme-stabilizing reagent, in addition to trehalose, may have been involved unintentionally in the first study. One significant difference between the two studies was that the devices in the first study were fabricated via photolithography using an SU-8 photoresist and the devices in the third study were fabricated via wax printing. ${ }^{10,24} \mathrm{We}$ hypothesized that the technique used to fabricate the devices may have had an influence on the stability of the enzymes and, more specifically, that some residual SU-8 on the paper contributed to an increased stability of the enzymes in the first study. Therefore, we were interested in studying the effects of SU-8 and trehalose independently on the stability of HRP.

SU-8 is the main component of SU-8 photoresists, which are used extensively for microfabrication and allow for structures with high aspect ratios to be patterned on solid substrates. ${ }^{25}$ The interaction of SU-8 and enzymes has been explored in the context of preparing microfluidic sensors. ${ }^{26-29}$ Enzymes including GOx, lactate oxidase, choline oxidase, and glutamate oxidase were immobilized on SU-8 structures by simply applying solutions of the enzymes to the structures and allowing the solutions to dry under ambient conditions. ${ }^{27-29}$ The authors of these studies hypothesized that some residual, unreacted epoxy groups from SU-8 on the surface of the SU-8 structures were reacting with amine or carboxylate groups from the enzymes to covalently bond the enzymes to SU-8. In a different study, poly(ethylene glycol) diglycidyl ether (PEGDE), which contains two epoxy groups, was used to immobilize enzymes on a platinum electrode, and the authors showed that the immobilized enzymes had extended shelf-lives and sensitivities that were comparable to enzymes that were fixed on the electrodes using glutaraldehyde. ${ }^{30}$ It is possible that SU-8 could stabilize enzymes through a similar mechanism as PEGDE and glutaraldehyde by forming covalent bonds to an enzyme and thereby stabilizing the enzyme's tertiary and quaternary structures. ${ }^{30-33}$ The study involving immobilization of GOx on SU-8 structures monitored the sensitivity of the devices over the course of 49 days and observed a significant decrease in the sensitivity of the devices during the first 10 days, presumably due to degradation of the enzyme, but no control experiments were performed to determine whether immobilizing enzymes on SU-8 had a stabilizing effect. ${ }^{27}$

One of the challenges with studying the stability of enzymes is that these studies are typically conducted over the course of several weeks or months and require a significant investment of time and resources to complete. So, a final objective of this project was to establish a method for quickly evaluating the effect of reagents on the stability of enzymes. To do so, we set up two experiments: a short-term study, where we measured the activity of various concentrations of HRP within $1 \mathrm{~h}$ of drying the HRP solutions on paper, and a long-term study, where we measured the activity of one concentration of HRP dried and stored on paper over the course of 2 months. By comparing the results of these two studies, we planned to 
identify results from the short-term study that could be useful for predicting the results of the long-term study.

\section{MATERIALS AND METHODS}

Materials and Reagents. All materials and reagents were obtained from commercial sources unless otherwise stated. The following materials and reagents were used: chromatography paper (Whatman grade $1 \mathrm{Chr}, 20 \mathrm{~cm} \times 20 \mathrm{~cm}$, GE Healthcare Life Sciences), solid ink (Xerox), HRP (293 U/mg, EMD Millipore), D-(+)-trehalose dihydrate (trehalose, 99\%, ACROS Organics), EPON resin SU-8 (SU-8, Momentive), 1-step ABTS (a proprietary HRP substrate solution, Thermo Scientific), $1 \times$ phosphate-buffered saline ( $1 \times \mathrm{PBS} \mathrm{pH} 7.4$, prepared from a $10 \times$ solution, Fisher BioReagents), acetonitrile (HPLC grade, Fisher Chemical), 2-methylpropan-2-ol (tert-butyl alcohol, 99\%, Alfa Aesar), erioglaucine sodium salt (blue dye, Alfa Aesar), tartrazine (yellow dye, Alfa Aesar), nanopure-filtered water, heat-seal bags (Foodsaver), silica gel desiccant packets (1 g, Dry \& Dry), and aluminum foil (Reynolds).

Solution Preparation. A stock solution of HRP (182 U/ $\mathrm{mL}$ ) was prepared in $1 \times$ PBS. A stock solution of trehalose $(34.2 \% \mathrm{w} / \mathrm{v}$ or $34.2 \mathrm{~g} / \mathrm{dL})$ was prepared in nanopure water, and a stock solution of SU-8 $(10.0 \% \mathrm{w} / \mathrm{v}$ or $10.0 \mathrm{~g} / \mathrm{dL})$ was prepared in a solvent mixture composed of $30 \%$ tert-butyl alcohol and $70 \%$ acetonitrile by volume. These stock solutions were further diluted in their respective solvents to prepare working solutions. All solutions were prepared accurately using an analytical balance, volumetric flasks, volumetric pipets, and micropipets. The HRP stock solution was stored at $4{ }^{\circ} \mathrm{C}$ in aliquots. The trehalose and SU-8 stock solutions were stored at room temperature in glass bottles. A green dye solution containing erioglaucine $(2 \mathrm{mM})$ and tartrazine $(15 \mathrm{mM})$ was prepared in nanopure water.

Fabrication of Paper Microzone Plates. Paper microzone plates were fabricated via wax printing. ${ }^{24}$ The plates were designed in CleWin (version 2.89) using the same layout as conventional plastic 96-well plates (Figure 1B). The microzones were designed as circular yellow rings with a diameter of $6.00 \mathrm{~mm}$ and a line width of $0.30 \mathrm{~mm}$. The design was then saved as a postscript file and printed from Adobe Illustrator onto chromatography paper using a solid ink printer (Xerox Phaser 8560) set to "photo" print quality. The printed sheets of paper were heated in a compact forced air convection oven (MTI Corporation) set to $175{ }^{\circ} \mathrm{C}$ for $6 \mathrm{~min}$ and were then cooled to room temperature under ambient conditions. The final diameter of the microzones was $4.6 \mathrm{~mm}$, and they could be filled with $1.0 \mu \mathrm{L}$ of solution. The microzone plates were cut out using scissors and stored wrapped in aluminum foil until they were used.

Dispensing Solutions on Paper Microzone Plates Using a Mantis Liquid Handling Robot. Solutions were dispensed onto the paper microzone plates using a Mantis liquid handling robot (Formulatrix, Inc.). A custom-made manifold was used to hold the paper microzone plates on the Mantis in the correct position for dispensing (Figure S1). Trehalose solutions, HRP solutions, and $1 \times$ PBS were dispensed using low-volume $(0.1-0.5 \mu \mathrm{L})$ silicone chips. Solutions of SU-8 were dispensed using low-volume perfluoroelastomer chips. The reagent solution for the HRP colorimetric activity assay (1-step ABTS) was dispensed using a high-volume $(1-5 \mu \mathrm{L})$ silicone chip.

An initial experiment was performed to investigate the feasibility of preparing dilutions of a stock HRP solution directly on the paper microzone plates. The Mantis was loaded with a $41.9 \mathrm{U} / \mathrm{mL}$ solution of HRP and $1 \times$ PBS and was programmed to dispense these two solutions in various proportions. When it was programed to dispense two different solutions, the Mantis will dispense the first solution in all of the indicated zones and will then dispense the second solution. Therefore, we tested depositing the HRP stock solution first followed by $1 \times$ PBS, and we also tested depositing the $1 \times$ PBS first followed by the HRP stock solution. For comparison, we also prepared a 1:1 mixture of the HRP stock solution and $1 \times$ PBS manually. Twelve replicates of each dilution were prepared on three separate microzone plates. After depositing the corresponding solutions, the plates were dried for 30 min under ambient conditions and then an activity assay for HRP was performed as described below.

Activity Assay for HRP on Paper Microzone Plates. A colorimetric activity assay was performed on the paper microzone plates to determine the activity of the HRP present in each microzone. ${ }^{16}$ To perform the assay, $3 \mu \mathrm{L}$ of 1 -step ABTS solution at room temperature was deposited in each microzone, and the reaction was allowed to proceed for $30 \mathrm{~min}$ under ambient conditions. After $30 \mathrm{~min}$, the microzones were completely dry, and the intensity of the color in each zone was quantified via digital image colorimetry (DIC; Figure S2). ${ }^{23}$ The plates were scanned using a flatbed scanner (Epson Perfection V300, 48-bit color, 300 dpi resolution), and the images were analyzed in Image $1.46 \mathrm{r}$. First, the images were inverted; then, they were split into the red, green, and blue color channels. The mean pixel intensity of each microzone was measured in the red channel using a microarray profile plugin. ${ }^{34}$ The entire area of each microzone was selected for the analysis (Figure S2B). The mean intensity values were analyzed in Excel and Kaleidagraph.

Effect of Trehalose and SU-8 on the Stability of HRP. The effect of trehalose and SU-8 on the stability of HRP was studied in two different contexts: a short-term study, where the activities of various concentrations of HRP dried on paper microzone plates were measured after $1 \mathrm{~h}$, and a long-term study, where the activity of a single concentration of HRP dried and stored on paper microzone plates was monitored over the course of 61 days.

For the short-term study, solutions of trehalose and SU-8 with concentrations of $0,0.1,0.5,1,5$, and $10 \% \mathrm{w} / \mathrm{v}$ were tested; for $0 \%$ trehalose, we used nanopure water, and for $0 \%$ SU-8, we used a solvent mixture of $30 \%$ tert-butyl alcohol and $70 \%$ acetonitrile by volume. Up to three paper microzone plates for each concentration of stabilizing reagent were prepared by depositing $1 \mu \mathrm{L}$ of stabilizing reagent solution in all 96 microzones and drying the plates overnight. A green dye solution $(2 \mu \mathrm{L})$ was added manually to the microzones in the four corners of the plate (zones A1, A12, H1, and H12) to aid image analysis of the plates in ImageJ (Figure S2). On the day of the experiment, five stock solutions of HRP with concentrations of $182,18.2,1.82,0.182$, and $0.0182 \mathrm{U} / \mathrm{mL}$ were prepared from a $182 \mathrm{U} / \mathrm{mL}$ stock solution by serial dilution in $1 \times$ PBS. The HRP solutions were loaded onto the Mantis along with $1 \times$ PBS, and these solutions were dispensed in various proportions onto the microzones containing the dried stabilizing reagents as described in Table S1. For each plate, $1 \times$ PBS was dispensed first followed by the corresponding HRP solutions. Through this process, we generated a range of concentrations of HRP from 0.00319 to $182 \mathrm{U} / \mathrm{mL}$ in the microzones. A total volume of $4 \mu \mathrm{L}$ was 
dispensed in each microzone; therefore, the total quantity of HRP deposited in each microzone was in the range of $0.0127-$ $782 \mathrm{mU}$. The HRP was deposited on the microzone plates in the order of the lowest concentration of stabilizing reagent to the highest and in the order of the lowest concentration of HRP stock solution to the highest. The plates were then allowed to dry for $1 \mathrm{~h}$ under ambient conditions, and the HRP activity assay was performed as described above. The experiments using trehalose and SU-8 were performed on separate days, and in a third experiment, a $34 \%$ trehalose solution was also tested following the same procedure. The average mean intensity value from the background zones (columns 1 and 12 , rows $B-G$ ) was subtracted from the mean intensity values of all other zones on the plate to give the background-corrected mean intensities. An example of a completed paper microzone plate is shown in Figure S2.

For the long-term study, 34\% trehalose, $1 \%$ SU-8, and a control with no enzyme-stabilizing reagent were tested. Twenty-two paper microzone plates were prepared on day 0 of the experiment. The stabilizing reagents $(1 \mu \mathrm{L})$ were deposited in each microzone; they were allowed to dry for at least $1 \mathrm{~h}$ under ambient conditions, and then $4 \mu \mathrm{L}$ of either a $31.9 \mathrm{U} / \mathrm{mL}$ HRP solution or $1 \times$ PBS was deposited in each zone as described in Table S2. The HRP solutions were allowed to dry for $1 \mathrm{~h}$, and then 11 plates were vacuum sealed in a plastic pouch along with a silica gel desiccant packet (Figure S3). The remaining 11 plates were wrapped individually in aluminum foil. All plates were stored in a drawer under ambient conditions. One plate from each set was tested using the HRP activity assay on days $0,1,2,3,7,14,21,28,35$, and 61 . The ambient temperature during the experiment fluctuated between 18 and $24{ }^{\circ} \mathrm{C}$, and the ambient relative humidity fluctuated between 36 and 52\%.

Data Analysis and Calculations. The results from the short-term experiment produced calibration curves in the form of mean intensity (I) versus quantity of HRP ( $Q$ ) for each concentration of stabilizing reagent (Figure 2). The shape of these calibration curves can be modeled using the following equation

$$
I=\frac{I_{\max } \times Q}{K+Q}
$$

where $I_{\max }$ is the maximum mean intensity and $K$ is the quantity of HRP required to produce a mean intensity equal to half of $I_{\max }{ }^{23,35}$ Fitting each calibration curve with eq 1 in Kaleidagraph gave the magnitudes of $I_{\max }$ and $K$ for each concentration of stabilizing reagent.

To quantify and compare the effects of the various concentrations of trehalose and SU-8 on the stability of HRP in the short-term study, we defined the stabilization factor based on $K\left(\mathrm{SF}_{K}\right)$ as the following ratio

$$
\mathrm{SF}_{K}=\frac{K_{0 \%}}{K_{x \%}}
$$

where $K_{0 \%}$ is the magnitude of $K$ in the presence of no (0\%) stabilizing reagent and $K_{x \%}$ is the magnitude of $K$ in the presence of any other $(x \%)$ concentration of stabilizing reagent. This way, lower values of $K_{x \%}$, which are indicative of greater stabilization of the HRP, correlate with larger stabilization factors.

As alternatives to $\mathrm{SF}_{K}$, we also defined two other stabilization factors: stabilization factor based on the quantity of $\mathrm{HRP}\left(\mathrm{SF}_{\mathrm{Q}}\right)$
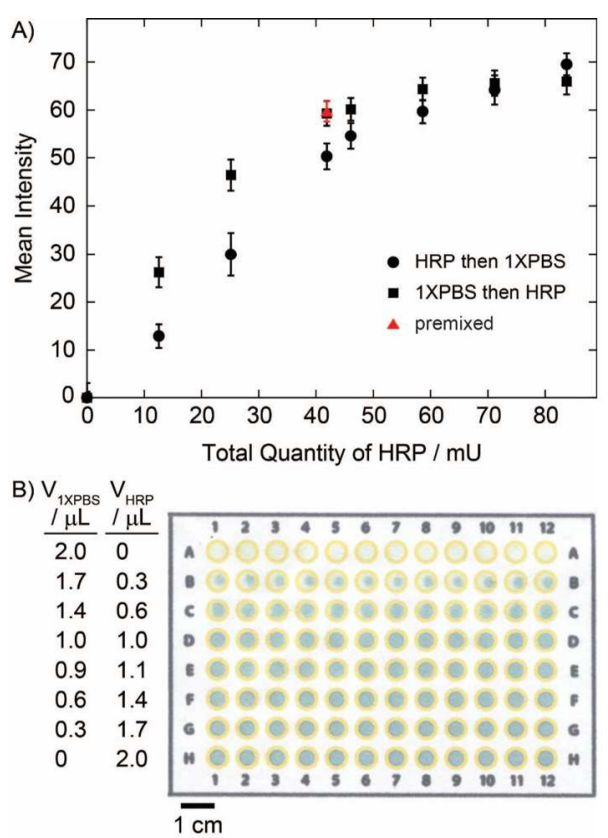

Figure 2. Dilution of HRP stock solutions on paper microzone plates. (A) Plot of background-corrected mean intensities measured from the microzones versus the total quantity of HRP deposited in each microzone. The HRP dilutions were prepared directly on paper microzone plates from a single HRP stock solution with either the HRP stock solution or the diluent buffer ( $1 \times$ PBS) being deposited first. The HRP stock solution and $1 \times$ PBS were also premixed manually and deposited in microzones for comparison. Deposition of the diluent buffer first followed by the stock solution of HRP produced more accurate results when compared to the dilution that was prepared manually. Data points represent the mean of 12 replicates and error bars represent one standard deviation from the mean. (B) Image of the paper microzone plate where $1 \times$ PBS was deposited first. The volumes of $1 \times$ PBS and HRP stock solution deposited in each microzone are given. Volumes of HRP below $0.6 \mu \mathrm{L}$ resulted in nonuniform distributions of color across the microzones; therefore, 0.7 $\mu \mathrm{L}$ was the smallest volume of HRP used in the stabilization experiments.

and stabilization factor based on the limit of detection $\left(\mathrm{SF}_{\mathrm{LOD}}\right)$. $\mathrm{SF}_{\mathrm{Q}}$ was calculated using the following equation

$$
\mathrm{SF}_{Q}=\frac{Q_{0 \% \text { eq }}}{Q_{x \%}}
$$

where $Q_{x \%}$ is the total quantity of HRP delivered to a microzone containing a given concentration $(x \%)$ of stabilizing reagent and $Q_{0 \% \text { eq }}$ is the theoretical quantity of HRP required to produce the same mean intensity as $Q_{x \%}$ when delivered to a microzone containing no $(0 \%)$ stabilizing reagent. The magnitude of $Q_{0 \% \text { eq }}$ was calculated using the following equation, which was derived from eq 1

$$
Q_{0 \% \text { eq }}=\frac{I_{x \%} \times K_{0 \%}}{I_{\max 0 \%}-I_{x \%}}
$$

where $I_{x \%}$ is the mean intensity obtained from $Q_{x \%}$, and $K_{0 \%}$ and $I_{\max } 0 \%$ are constants obtained by fitting the data for either the $0 \%$ trehalose or $0 \%$ SU-8 solution with eq 1 (Figure S4).

The limit of detection stabilization factor was calculated using the following equation 

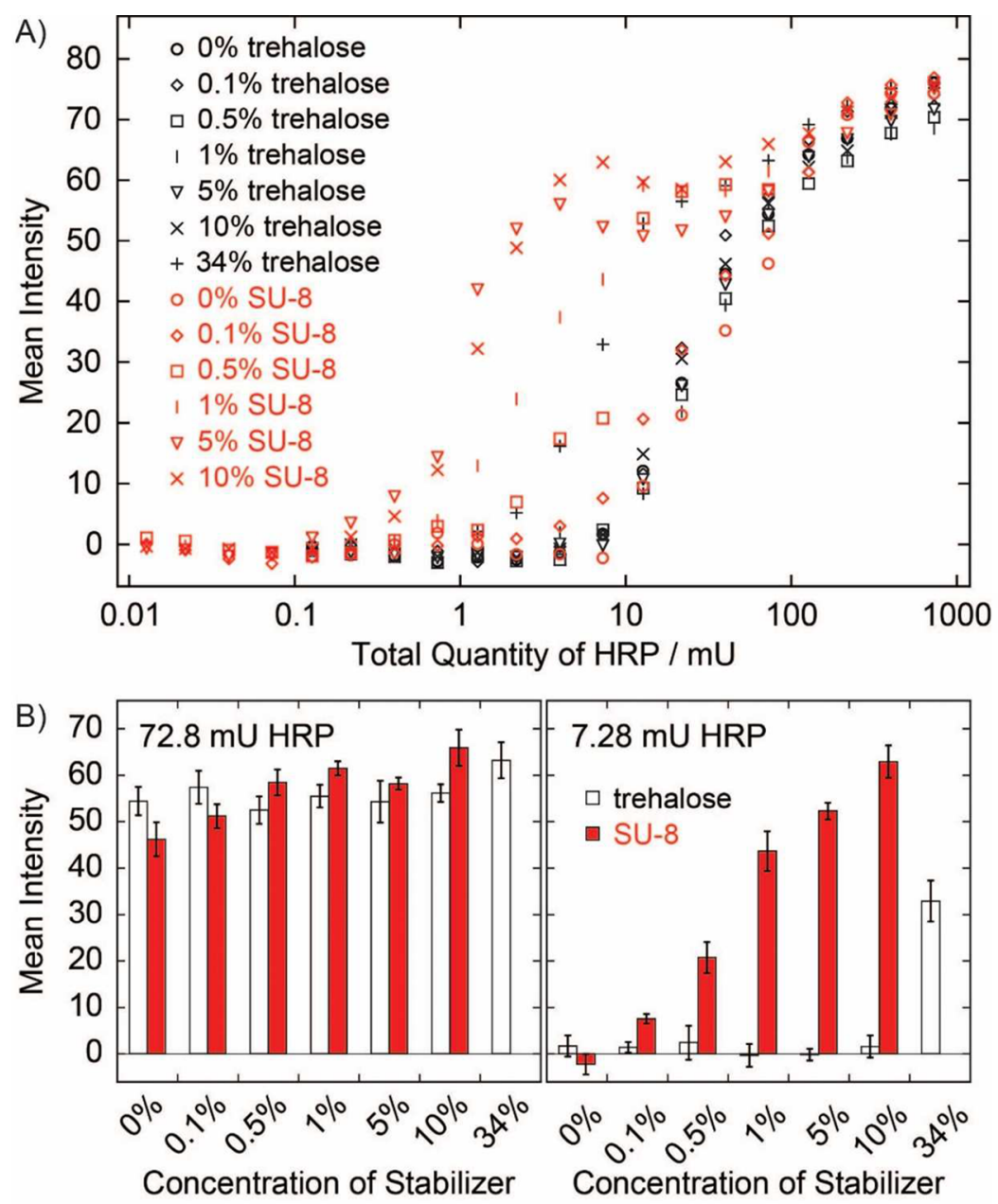

Figure 3. Short-term stabilization of HRP dried on paper in the presence of various concentrations of trehalose and SU-8. (A) Plot of the background-corrected mean intensities versus the total quantity of HRP deposited in each microzone. Data points represent the mean of 10 replicates; error bars were omitted for clarity but are shown in (B) for two quantities of HRP. Significantly higher signals were obtained from HRP dried in the presence of SU-8 compared to trehalose, which suggests that SU-8 is a more effective enzyme-stabilizing reagent. (B) Bar graphs of the background-corrected mean intensities for 72.8 and $7.28 \mathrm{mU}$ of HRP dried on paper in the presence of various concentrations of trehalose and SU-8. The height of the bars represents the mean of 10 replicates, and the error bars represent one standard deviation from the mean. A concentrationdependent response is observed at $7.28 \mathrm{mU}$ of HRP, where higher concentrations of stabilizing reagent tend to correlate with higher signals.

$$
\mathrm{SF}_{\mathrm{LOD}}=\frac{\mathrm{LOD}_{0 \%}}{\mathrm{LOD}_{x \%}}
$$

where $\mathrm{LOD}_{0 \%}$ is the minimum quantity of HRP required to generate a detectable signal when no $(0 \%)$ stabilizing reagent is present and $\mathrm{LOD}_{x \%}$ is the minimum quantity of HRP required to generate a detectable signal in the presence of a given concentration $(x \%)$ of stabilizing reagent. A detectable signal was defined based on the IUPAC definition of a signal that is at least 3 times greater than the standard deviation of the blank; in this study, the mean standard deviation of the blank was 2 intensity units, so the lowest detectable signal was defined as 6 intensity units. The limit of detection was then calculated for each concentration of stabilizing reagent by fitting the two data points with signals closest to 6 (one higher and one lower) with a linear trend line and using the equation from this line to calculate the amount of HRP required to produce a signal of 6 .

For the long-term study, we calculated the half-life $\left(t_{1 / 2}\right)$ of the signal for each experimental condition using a first-order exponential decay model (Figure S5). ${ }^{3}$

\section{RESULTS AND DISCUSSION}

The use of the Mantis for dispensing solutions onto paper microzone plates proved to be an efficient way of performing a large number of assays on paper. In total, the results from over 5000 assays are presented in this article, and many more tests were performed while we were optimizing the conditions for the different experiments. The Mantis can deliver a single solution to all 96 microzones in approximately $2 \mathrm{~min}$, depending on the volume that is dispensed and the chip that is used. While the Mantis is a convenient tool for conducting these experiments, the same experiments can also be performed manually, using pipets. We performed several preliminary experiments manually and obtained comparable results (data not shown).

When evaluating the order in which solutions were deposited with the Mantis for diluting stock solutions directly on the microzone plates, we found that the best results were obtained when the $1 \times$ PBS (diluent) was deposited first in the microzones followed by the HRP stock solution (Figure 2). The signal from the manual dilution was identical to the signal from the dilution prepared using the Mantis when $1 \times$ PBS was 
deposited first (Figure 2A). The signals obtained when the HRP stock solution was deposited first were significantly lower than the signals obtained when $1 \times$ PBS was deposited first, and this difference became more pronounced at lower concentrations of HRP. One possible explanation for this result is that the HRP stock solution begins to dry out immediately after it is deposited in a microzone, and it takes $\sim 2$ min before the $1 \times$ PBS is added to the microzone to dilute the HRP. Therefore, when the HRP is deposited first, it effectively goes through two drying cycles, which likely negatively impacts the activity of the HRP in the microzone. As would be expected, this effect became more pronounced as the volume of deposited HRP stock solution decreased since proportionally more of the HRP stock solution dried out before the $1 \times$ PBS was added.

An additional important result from this initial experiment is that the volume of the stock solution deposited in each zone should be at least $0.6 \mu \mathrm{L}$ in order to achieve uniform mixing and distribution of the reagent in the microzone when diluting stock solutions directly on a paper microzone plate (Figure 2B). Volumes of HRP stock solution below $0.6 \mu \mathrm{L}$ did not appear to mix sufficiently with the $1 \times$ PBS and resulted in nonuniform color distributions in the microzones. When volumes of HRP stock solution of $0.6 \mu \mathrm{L}$ or higher were deposited, a uniform color was observed in the test zones, which is desirable for more accurate quantification of the color intensity. This result represents one potential limitation of working with paper microzone plates. The maximum volume of water that can be delivered to a microzone and then dried out within $30 \mathrm{~min}$ is $\sim 10 \mu \mathrm{L}^{21}$ Under these conditions, the maximum dilution that could be achieved in a microzone is only a factor of 16 . The significance of this limitation is that to prepare a wide range of concentrations of a reagent, as was the case for this study, multiple stock solutions have to be prepared manually and then further diluted on the paper microzone plates using the Mantis.

To confirm that the results from the HRP stabilization experiments were not influenced by the solvents used to prepare the SU-8 and trehalose solutions or by changes in the activity of the HRP stock solution over time, we compared the results from the short-term study for the 0\% SU-8 and 0\% trehalose solutions collected on different days (Table S3). A two-tailed paired $t$-test confirmed that there were no statistically significant differences between the results for the two solvents collected both 4 and 7 days apart $(p=0.23$ and 0.56 , respectively). These results suggest that the two solvents evaporate completely from the microzones and have no effect on the activity of HRP and that the HRP stock solution can be stored at $4{ }^{\circ} \mathrm{C}$ without any significant change in activity for at least 7 days.

The complete results from the short-term stabilization study are shown in Figure 3A, where the activity of various quantities of HRP spanning 5 orders of magnitude were evaluated in the presence of various concentrations of trehalose and SU-8. At high quantities of HRP, the signal from the activity assay becomes saturated, and no significant differences are observed between the results for the various concentrations of trehalose and SU-8. As the quantity of HRP deposited in each microzone decreases, the signals also begin to decrease, and significant differences between the various concentrations of the two enzyme-stabilizing reagents can be observed (Figure 3B). Eventually, at sufficiently low quantities of HRP, no detectable signal is obtained from the activity assay. The results show that in the presence of SU-8, HRP produces significantly higher signals across a range of HRP quantities compared to HRP dried in the presence of trehalose. For example, $12.4 \mathrm{mU}$ of HRP was required in each microzone to produce a detectable signal in the presence of $10 \%$ trehalose, whereas $0.728 \mathrm{mU}$ of HRP was sufficient to provide a detectable signal in the presence of 5 and $10 \%$ SU-8. These results suggest that SU-8 is more effective than trehalose at stabilizing HRP dried on paper. The results also suggest that, by adding an appropriate enzymestabilizing reagent, it may be possible to use lower quantities of enzymes when preparing paper-based assays.

For SU-8, we observed a concentration-dependent response where the signal from a given quantity of HRP increased with increasing SU-8 concentration (Figure 3B), although quantities of HRP below $4.00 \mathrm{mU}$ produced the highest signals in the presence of $5 \%$ SU-8. Since SU-8 is hydrophobic, there is a limit to the amount of SU-8 that can be deposited on paper before making the paper completely hydrophobic. Although we were able to perform assays using the 5 and $10 \%$ SU-8 solutions, the microzones containing these higher concentrations of SU-8 wicked aqueous samples much more slowly than the microzones containing lower concentrations of SU-8. Therefore, we selected a concentration of $1 \%$ SU- 8 for the long-term study since this concentration showed significant stabilization of HRP in the short-term study and did not affect the hydrophilicity of the microzones to a significant extent.

For trehalose, a small stabilization effect was observed for the $0.1-10 \%$ solutions compared to the $0 \%$ control, but no significant differences were observed between the results from the various trehalose concentrations. One possible explanation for this result is that the paper microzone plates were prepared and tested in the order of the lowest concentration of trehalose to the highest. Thus, for any given quantity of HRP, the $0 \%$ trehalose experiments were performed approximately $15 \mathrm{~min}$ before the $10 \%$ trehalose experiments, and it is possible that the HRP stock solutions lost some activity while sitting on the Mantis during this time. To demonstrate that trehalose has a concentration-dependent stabilizing effect on HRP, we performed an additional test using a 34\% (1 M) trehalose solution, which did show more significant stabilization compared to that with $0.1-10 \%$ trehalose solutions. The solubility of trehalose in water is $\sim 1.5 \mathrm{M}$ at $20^{\circ} \mathrm{C},{ }^{36-38}$ but concentrations of trehalose above $1 \mathrm{M}$ were not tested in the short-term experiments because we wanted to ensure that trehalose would not precipitate in the liquid handling robot during deposition of the trehalose solutions. We selected the $34 \%$ trehalose solution for the long-term study since we expected this higher concentration of trehalose to have a more significant stabilizing effect on HRP compared to that of $10 \%$ trehalose, which we had already tested previously. ${ }^{16}$

The results shown in Figure 3 also demonstrate that to fully characterize the effect of a particular enzyme-stabilizing reagent on an enzyme it may be necessary to perform activity assays over a wide range of concentrations of the enzyme. For example, in the case of SU-8 and trehalose, the signal intensities varied significantly over 4 orders of magnitude of quantities of HRP. However, if the objective is only to identify potential enzyme-stabilizing reagents, then it should be possible to perform assays using a single quantity of HRP and look for an increase in the signal for HRP in the presence of the reagent compared to that of unstabilized HRP. A quantity of HRP in the range of 5-15 $\mathrm{mU}$, for this particular experimental design, would be the optimal quantity for performing an initial screening of potential enzyme-stabilizing reagents. Promising 
candidates from the initial screening could then be evaluated over a larger range of quantities of HRP to characterize the effects of the reagent on the activity of HRP in more detail.

The magnitudes of the three different stabilization factors $\left(\mathrm{SF}_{K}, \mathrm{SF}_{\mathrm{Q}}\right.$, and $\left.\mathrm{SF}_{\mathrm{LOD}}\right)$ calculated for each concentration of stabilizing reagent are shown in Figure 4. These stabilization

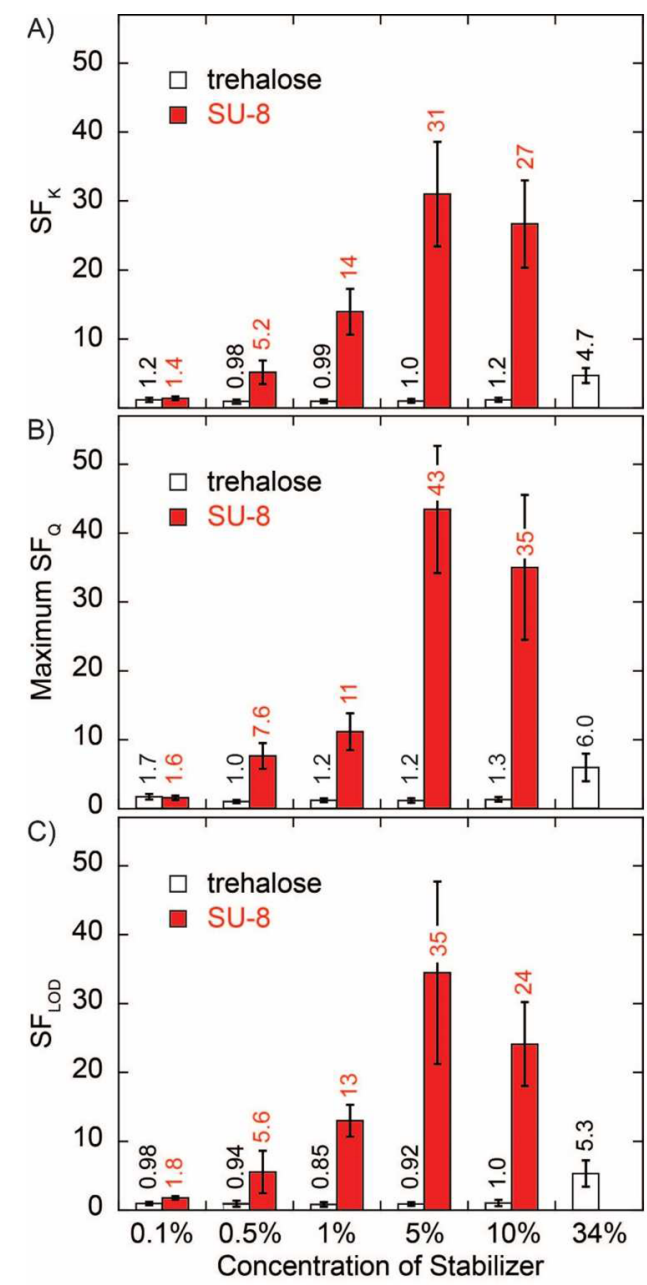

Figure 4. Bar graphs of the three stabilization factors calculated for the various concentrations of trehalose and $\mathrm{SU}-8: \mathrm{SF}_{K}(\mathrm{~A}), \mathrm{SF}_{Q}(\mathrm{~B})$, and $\mathrm{SF}_{\mathrm{LOD}}(\mathrm{C})$. The height of the bars represents the calculated stabilization factors, and the error bars represent the propagated uncertainty. For $\mathrm{SF}_{Q}$, only the maximum stabilization factors are shown for each concentration of stabilizing reagent. A complete plot of $\mathrm{SF}_{Q}$ 's for every quantity of HRP is shown in Figure S4. The stabilization factors serve as a simple quantitative way of comparing the effect of the various concentrations of stabilizing reagents on the stability of HRP. The results indicate that SU-8 can stabilize HRP better than trehalose and that, in general, higher concentrations of enzyme-stabilizing reagent result in greater stabilization of HRP for the concentrations that were tested.

factors provide a simple way of comparing the effect of the stabilizing reagents on the activity of HRP. All three graphs show similar trends and suggest that SU-8 is a much more powerful stabilizing reagent for HRP than trehalose. The 5\% SU-8 solution had the highest stabilization factor of all of the conditions that were tested, with magnitudes that were $\sim 35$ times larger than the respective stabilization factors determined for $5 \%$ trehalose. When comparing the stabilization factors for the $1 \%$ SU- 8 solution and the $34 \%$ trehalose solution, the two solutions that were tested in the long-term study, we found that the $1 \%$ SU- 8 solution gave stabilization factors that were, on average, 2.4 times larger than the stabilization factors for $34 \%$ trehalose.

For future studies, we believe the stabilization factors can serve as a simple way of comparing the effects of various stabilizing reagents or various concentrations of one stabilizing reagent quantitatively. We introduce the three different stabilization factors to allow for some flexibility in the experimental design of future studies, but we expect that only one stabilization factor would be used in any given study. Since $\mathrm{SF}_{K}$ is calculated by fitting all of the data points in a curve, it is likely going to be the most precise of the three stabilization factors and the least affected by indeterminate errors in any given data point. The disadvantage of $\mathrm{SF}_{K}$ is that it requires a complete calibration curve for each concentration of reagent as well as a calibration curve with no added reagent. However, these calibration curves could be generated with as few as four or five different concentrations of enzyme, albeit in an appropriate range, which could be determined from a preliminary experiment. The advantage of $\mathrm{SF}_{Q}$ is that it can be calculated from the results of a single experiment for each concentration of reagent and a calibration curve with no added reagent. However, as the data in Figure S4C shows, the magnitude of $\mathrm{SF}_{Q}$ can vary significantly as a function of the concentration of enzyme, so it would be important to optimize the concentration of the enzyme for the experiments. The advantages of $\mathrm{SF}_{\mathrm{LOD}}$ are that it can be calculated without having to prepare complete calibration curves and, since it relies on results obtained using low concentrations of enzyme, the experiments will require less enzyme than would be required for calculating the other stabilization factors, which could be useful in situations where the enzyme is expensive or available only in limited quantities.

The results from the long-term study are shown in Figure 5. We chose to apply $127 \mathrm{mU}$ of HRP to each microzone because this quantity of HRP produced a signal that was just below saturation, so it made it possible to track the loss of HRP activity over time. When no stabilizing reagent was added, the signal from the assay decayed rapidly to zero within 3 days, as observed previously. ${ }^{16}$ In the presence of $34 \%$ trehalose, the signal decayed more slowly but still reached zero by day 61 . In the presence of $1 \%$ SU-8, the signal decayed the slowest, and some detectable activity remained on day 61 . When the plates were vacuum sealed, a significant increase in the stability of the HRP was observed for all conditions. By day 61 , the signal from HRP stored with $1 \%$ SU- 8 decreased by only $10 \%$ compared to the signal on day 0 . The signal from HRP stored in the presence of $34 \%$ trehalose decreased by $\sim 50 \%$ by day 61 , whereas HRP stored with no stabilizing reagent lost most of its activity within 14 days, but some residual activity remained through day 61.

The half-lives of the signals from the long-term study are shown in Table 1 and provide a simple way of comparing the effects of the different stabilizing reagents and storage conditions on the stability of HRP. The half-lives of the HRP assay signal in the presence of $1 \%$ SU- 8 were 2.2 and 2.1 times longer than the half-life in the presence of $34 \%$ trehalose for the microzone plates stored in aluminum foil and stored vacuum sealed, respectively. Vacuum sealing the devices increased the half-lives of the signals by factors of 7.8 and 7.4 for $34 \%$ trehalose and $1 \%$ SU-8, respectively. These results suggest that enzyme-stabilizing reagents and the storage conditions 


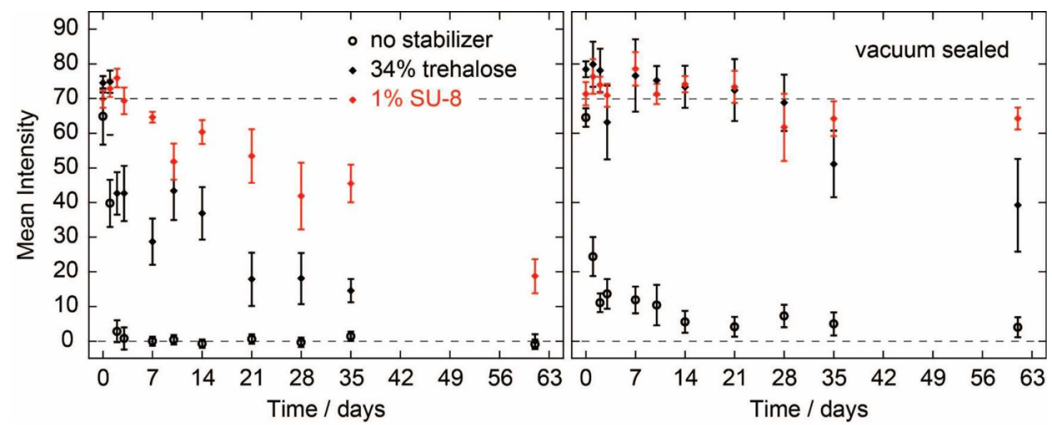

Figure 5. Long-term stabilization of HRP stored on paper microzone plates that were either wrapped in aluminum foil (left) or vacuum sealed in plastic (right) for up to 61 days. Data points represent the background-corrected mean intensity of 12 replicates, and error bars represent one standard deviation from the mean. The upper dashed line represents the mean signal from all microzones on day 0 . The lower dashed line represents a background-corrected mean intensity of zero. HRP had the highest stability when stored vacuum sealed in the presence of $1 \%$ SU-8.

Table 1. Half-Life of the Signal from the Activity Assay for HRP Stored under Various Conditions

\begin{tabular}{lcc} 
& \multicolumn{2}{c}{ half-life (days) } \\
\cline { 2 - 3 } stabilizing reagent & aluminum foil & vacuum sealed \\
none & 0.44 & 5.1 \\
$34 \%$ trehalose & 14 & 109 \\
$1 \%$ SU-8 & 31 & 229 \\
\hline
\end{tabular}

influence the stability of HRP independently and confirm the results from previous studies showing that storing enzymes in a dry, vacuum sealed environment can extend their shelf life significantly. $^{8}$

By comparing the results of the short- and long-term experiments, we found that the both the half-lives and the stabilization factors for $1 \%$ SU-8 were approximately twice as large as the respective half-lives and stabilization factors for $34 \%$ trehalose. This result suggests that the stabilization factors could serve as a quick way of predicting the stabilizing effects of a reagent on an enzyme without having to conduct a long-term study, which could be useful for identifying and characterizing new enzyme-stabilizing reagents or for quickly comparing the enzyme-stabilizing effects of various reagents or various concentrations of one reagent.

\section{CONCLUSIONS}

Paper microzone plates were demonstrated as a tool for studying the stability of enzymes on paper, and a simplified version of the short-term stabilization experiment described in this article could be used as a standardized method for screening reagents for enzyme-stabilizing effects. By calculating the stabilization factors for potential enzyme-stabilizing reagents, it should be possible to quickly compare the effects of various types or concentrations of reagents on the stability of a particular enzyme. Reagents that show promising results in the initial screen could then be further tested in long-term studies to confirm the results of the short-term experiments.

The use of stabilizing reagents can enhance the activity of HRP significantly on paper-based devices and can extend the shelf life of HRP stored on paper under ambient conditions. SU-8 was shown to stabilize HRP much more effectively than trehalose when it was added to paper at low concentrations. Storing paper-based devices in vacuum sealed pouches containing a desiccant also significantly enhanced the stability of HRP. What we find exciting about the use of SU-8 as a stabilizing reagent for HRP on paper-based microfluidic devices is that dilute solutions of SU-8 could be added to the test zones of devices before the reagents for a particular assay are added, and this step would represent only a minimal increase in the work required to prepare the device, but it could lead to significant improvements in the shelf life of the device.

\section{ASSOCIATED CONTENT}

\section{S Supporting Information}

The Supporting Information is available free of charge on the ACS Publications website at DOI: 10.1021/acs.analchem.6b05111.

Experimental details (Figures S1-S3), calibration curves and results from the calculations of $\mathrm{SF}_{Q}$ (Figure S4), exponential fit of the long-term study results (Figure S5), description of the layout of the paper microzone plates (Tables S1 and S2), and results with no added stabilizing reagent (Table S3) (PDF)

\section{AUTHOR INFORMATION}

\section{Corresponding Author}

*E-mail: awmartin@calpoly.edu. ORCID ${ }^{\circ}$

Andres W. Martinez: 0000-0002-2696-9165

\section{Notes}

The authors declare no competing financial interest.

\section{ACKNOWLEDGMENTS}

This material is based on work supported by the National Science Foundation under Grant No. 1605499. This project was also supported by a Research, Scholarly, and Creative Activities grant from California Polytechnic State University, San Luis Obispo, an Undergraduate Analytical Research Program Grant from the Society for Analytical Chemists of Pittsburgh, a Non-Tenured Faculty Grant from 3M, and a seed grant from the Cal Poly Center for Applications in Biotechnology funded by the Chevron Biotechnology Applied Research Endowment. We would like to thank Scott Ganaja and Progressiv Engineering Inc. for assistance with fabricating the manifold. We would like to thank Derek Gragson, Kevin Dunham, and Lisa Liu for help with obtaining supplies and equipment.

\section{REFERENCES}

(1) van Beilen, J. B.; Li, Z. Curr. Opin. Biotechnol. 2002, 13 (4), 338344. 
(2) Iyer, P. V.; Ananthanarayan, L. Process Biochem. 2008, 43 (10), 1019-1032.

(3) Lu, S.; Wang, X.; Lu, Q.; Hu, X.; Uppal, N.; Omenetto, F. G.; Kaplan, D. L. Biomacromolecules 2009, 10, 1032-1042.

(4) Fernández-Lafuente, R.; Rodríguez, V.; Bastida, A.; Blanco, R. M.; Alvaro, G.; Guisán, J. M. Stud. Org. Chem. 1993, 47, 315-322.

(5) Colaco, C.; Sen, S.; Thangavelu, M.; Pinder, S.; Roser, B. Bio/ Technology 1992, 10 (9), 1007-1011.

(6) Carpenter, J. F.; Crowe, L. M.; Crowe, J. H. Biochim. Biophys. Acta, Gen. Subj. 1987, 923 (1), 109-115.

(7) Gibson, T. D.; Hulbert, J. N.; Woodward, J. R. Anal. Chim. Acta 1993, 279 (1), 185-192.

(8) Ramachandran, S.; Fu, E.; Lutz, B.; Yager, P. Analyst 2014, 139

(6), 1456-1462.

(9) Chin, C. D.; Linder, V.; Sia, S. K. Lab Chip 2007, 7, 41-57.

(10) Martinez, A. W.; Phillips, S. T.; Butte, M. J.; Whitesides, G. M. Angew. Chem., Int. Ed. 2007, 46, 1318-1320.

(11) Martinez, A. W.; Phillips, S. T.; Whitesides, G. M.; Carrilho, E. Anal. Chem. 2010, 82 (1), 3-10.

(12) Cate, D. M.; Adkins, J. A.; Mettakoonpitak, J.; Henry, C. S. Anal. Chem. 2015, 87, 19-41.

(13) Yetisen, A. K.; Akram, M. S.; Lowe, C. R. Lab Chip 2013, 13

(12), 2210-2251.

(14) Mabey, D.; Peeling, R. W.; Ustianowski, A.; Perkins, M. D. Nat. Rev. Microbiol. 2004, 2 (3), 231-240.

(15) Martinez, A. W.; Phillips, S. T.; Carrilho, E.; Thomas, S. W.; Sindi, H.; Whitesides, G. M. Anal. Chem. 2008, 80 (10), 3699-3707.

(16) Mitchell, H. T.; Noxon, I. C.; Chaplan, C. A.; Carlton, S. J.; Liu, C. H.; Ganaja, K. A.; Martinez, N. W.; Immoos, C. E.; Costanzo, P. J.; Martinez, A. W. Lab Chip 2015, 15, 2213-2220.

(17) Yagoda, H. Ind. Eng. Chem., Anal. Ed. 1937, 9 (2), 79-82.

(18) Lehmann, S.; Delaby, C.; Vialaret, J.; Ducos, J.; Hirtz, C. Clin. Chem. Lab. Med. 2013, 51 (10), 1897-1909.

(19) Frank, R. Tetrahedron 1992, 48 (42), 9217-9232.

(20) Blackwell, H. E. Curr. Opin. Chem. Biol. 2006, 10, 203-212.

(21) Carrilho, E.; Phillips, S. T.; Vella, S. J.; Martinez, A. W.; Whitesides, G. M. Anal. Chem. 2009, 81 (15), 5990-5998.

(22) Azevedo, A. M.; Martins, V. C.; Prazeres, D. M. F.; Vojinović, V.; Cabral, J. M. S.; Fonseca, L. P. Biotechnol. Annu. Rev. 2003, 9, 199247.

(23) Chaplan, C. A.; Mitchell, H. T.; Martinez, A. W. Anal. Methods 2014, 6, 1296.

(24) Carrilho, E.; Martinez, A. W.; Whitesides, G. M. Anal. Chem. 2009, 81 (16), 7091-7095.

(25) Abgrall, P.; Conedera, V.; Camon, H.; Gue, A. M.; Nguyen, N. T. Electrophoresis 2007, 28, 4539-4551.

(26) Psoma, S. D.; van der Wal, P. D.; Frey, O.; de Rooij, N. F.; Turner, A. P. F. Biosens. Bioelectron. 2010, 26 (4), 1582-1587.

(27) Talaei, S.; Frey, O.; Psoma, S.; Van Der Wal, P. D.; De Rooij, N. F. Procedia Eng. 2010, 5, 448-451.

(28) Talaei, S.; van der Wal, P. D.; Ahmed, S.; Liley, M.; De Rooij, N. F. Microfluid. Nanofluid. 2015, 19, 351-361.

(29) Thomas, N.; Lahdesmaki, I.; Parviz, B. A. Microsyst. Technol. 2011, 17 (9), 1505-1510.

(30) Vasylieva, N.; Barnych, B.; Meiller, A.; Maucler, C.; Pollegioni, L.; Lin, J. S.; Barbier, D.; Marinesco, S. Biosens. Bioelectron. 2011, 26 (10), 3993-4000.

(31) López-Gallego, F.; Betancor, L.; Mateo, C.; Hidalgo, A.; AlonsoMorales, N.; Dellamora-Ortiz, G.; Guisán, J. M.; Fernández-Lafuente, R. J. Biotechnol. 2005, 119 (1), 70-75.

(32) Ritter, D. W.; Newton, J. M.; McShane, M. J. RSC Adv. 2014, 4 (53), 28036

(33) Mateo, C.; Palomo, J. M.; Fernandez-Lorente, G.; Guisan, J. M.; Fernandez-Lafuente, R. Enzyme Microb. Technol. 2007, 40, 14511463.

(34) Dougherty, B.; Rasband, W. MicroArray Profile plugin for ImageJ; OptiNav, Inc: Bellevue, WA, 2012.

(35) Schonhorn, J. E.; Fernandes, S. C.; Rajaratnam, A.; Deraney, R. N.; Rolland, J. P.; Mace, C. R. Lab Chip 2014, 14 (24), 4653-4658.
(36) Lammert, A. M.; Schmidt, S. J.; Day, G. A. Food Chem. 1998, 61 (1-2), 139-144.

(37) Miller, D. P.; de Pablo, J. J.; Corti, H. Pharm. Res. 1997, 14, 578-590.

(38) Gharsallaoui, A.; Rogé, B.; Génotelle, J.; Mathlouthi, M. Food Chem. 2008, 106 (4), 1443-1453. 